International Journal of Advanced Trends in Computer Science and Engineering

Available Online at http://www.warse.org/IJATCSE/static/pdf/file/ijatcse1401032021.pdf

https://doi.org/10.30534/ijatcse/2021/1411032021

\title{
Towards Covid-19 Detection in X-ray images using Convolutional Neural Network
}

\author{
Marium Malik ${ }^{1}$, Tehreem Masood ${ }^{2}$, Maira Kamran³ \\ ${ }^{1}$ Dept. of Software Engineering, The Superior University, Lahore, Pakistan. maryammalick2@ gmail.com \\ ${ }^{2}$ Dept. of Software Engineering, The Superior University, Lahore, Pakistan. tehreem.masood5@gmail.com \\ ${ }^{3}$ Dept. of Software Engineering, The Superior University, Lahore, Pakistan. lanamarii7@ gmail.com
}

\section{ABSTRACT}

Over the past 18 months, COVID-19 is continuously causing deadly consequences and devastating economic situations around the world. The vaccinations are still not adequately mature and vaccinating all the people around the world is a long process. Moreover, there is a rapid generation of variants of COVID-19 that are difficult to handle promptly. The skyrocketing pandemic of COVID-19 has knocked down the regular lifestyle of everyone, from individuals to massive corporations. The health industry is overburdened by the increasing number of patients that they are receiving. Efficient and stable screening methods of this disease will help in speeding up the detection process and take appropriate measures to flatten the curve on this disease. There is a high demand for COVID-19 discovery frameworks where the existing approaches still have some drawbacks. The most commonly used methods such as RTPCR have drawbacks of high false-positive rates, costly special kits, providing delayed and less accurate test results. Therefore, other diagnostic methods are actively explored. In this scenario, Artificial Intelligence comes to utilization with its branch of deep learning. Convolutional neural networks (CNNs) are a class of deep learning that are utilized in analyzing images, including medical imaging like Chest X-rays. This paper aims to analyze the power brought by CNNs to medical imaging in solving the global problem of early identification of COVID-19 in potential patients by analyzing their X-ray scans. Moreover, the entire process of image acquisition and analysis, its positive impact by minimizing asymptomatic patient contact with others and increasing the efficiency of the work environment at the frontline hospitals is discussed. The test results in this study showed that an accuracy of $95.11 \%$ was obtained on a dataset of 2905 images and an accuracy of $96.07 \%$ was obtained on an augmented dataset of 9337 images to detect Covid-19 in X-ray images, thus proving that deep learning models work better if the size of the training dataset is increased.

Key words: Convolutional Neural Networks, Deep Learning, CNN, COVID-19, Artificial Intelligence.

\section{INTRODUCTION}

A mysterious type of respiratory illness was initially reported to the WHO country headquarters from Wuhan City at the Hubei Province, China on the 31st of December, 2019. The analysis showed its similarity with viral pneumonia and the patients suffering from it showed symptoms of fever, cough, tiredness, and more. WHO declared this epidemic as an international public health emergency on the 30th of January 2020. They referred to it as COVID-19 on the 11th of February 2020 [1]. Another name of COVID-19 is- Severe Acute Respiratory Syndrome Coronavirus 2 (SARS-CoV-2). Although in mild cases COVID-19 causes' respiratory illness like fever and cough, in acute cases it triggers severe soreness in the air pods of the lungs that triggers trouble in oxygen exchange [2]. In various cases, several other symptoms are also witnessed such as the formation of mucus, mild to medium headache, hemoptysis, looseness of the bowels, and dyspnea [3]. The novel Coronavirus 2019, commonly known as COVID-19 has spread exponentially throughout the globe. Since its discovery at Wuhan, China in late December 2019, the current toll of active cases has reached 4,619,477 (as per World Health Organization statistics) and the death toll has reached 311,847 worldwide. Researchers are actively working on devising the most efficient mechanism to automatically screen COVID-19 patients. There is a critical requirement to identify patients who possess COVID-19 at the earliest so that the spread of this contagious disease can be controlled effectively and the great stress on the healthcare environment can be reduced. The reverse Transcription Polymerase Chain Reaction (RTPCR) diagnostic technique is commonly applied globally as a standard test for the detection of Covid-19. However, due to the high influx of patients, a limited amount of test kits available in the majority of the countries that resulted in a delayed result provision, and the high false-positive result rate of RTPCR technique, other detection methods using medical imaging like deep learning techniques are worthy of exploration. Computed Tomography (CT) scans, that are utilized by arrangement and prediction of Corona Virus features demonstrated accurate results in predicting asymptomatic patients [4]. Research has also proven that the sensitivity of Chest CT is high as compared to the RT-PCR test [5]. Additionally, a Chest X-Ray (CXR) is a widely utilized radiograph for image test diagnosis. Chest radiographs (CXRs) are more useful for diagnosing several conditions affecting the chest [6]. Till now, several models have been suggested to detect COVID-19 in the Chest X-ray scans with the usage of image pre-processing and various deep learning techniques. Chest $\mathrm{X}$-ray scans are one of the most commonly captured medical image types, their machines are extensively available in the majority of the hospital settings, and the scans are comparatively cheaper than other types. Therefore, success in detecting COVID-19 from X-ray images will solve various other problems. In the current scenario chest, x-ray images are used for the diagnoses of COVID-19 through frameworks that utilize deep learning techniques [7], [8], [9]. These models can be used as an alternative to the PCR methods.
Convolutional Neural Networks (CNN) is a domain that is derived from Deep Learning, which is a branch of Machine Learning that works by extracting features and self-learning to recognize the input images. Machine Learning lies under the umbrella of Artificial Intelligence that is a term introduced by John McCarthy, in 1995, during the Dartmouth Conference which focuses on machines that possess cognitive abilities like humans [10]. A CNN comprises an input, output, and various hidden layers that convolve using multiplication and dot products. Convolutional Neural Network algorithms use convolving filters and multiple layers to extract and learn features from an image a large number of features can be extracted using a higher quantity of tiers [11].

Hyperparameter tuning and utilization of static vectors help to achieve more accurate results [12]. CNN architecture is divided into feature learning and classification sections. The Feature-learning portion extracts the features from an input image based on its convolutional layers and ReLU function, and pooling algorithms. The classification layers then take the features and classify them. The convolutional layer creates a feature map from the input images using filters that are two-dimensional matrices, instead of assigning any convolutional weights or weighted sums. Moreover, the pooling tier works to decrease the dimensions of the image. The workflow that a typical CNN works in, where it takes an input, applies feature learning and classification to identify the object in the input image.

This study aims to investigate the performance and accuracy of the recently proposed CNN models that detect COVID-19 disease by a thorough investigation of chest X-ray scans and state their level of success, limitations, and failure rate in doing so. The input parameters, architectural frameworks used for transfer learning, methodology, and the adaptability of the models will be reviewed. Furthermore, this paper analyzes how accurate the results of such frameworks are and how adaptable they are, by considering their drawbacks and advantages.

The central aims of this study are as follows:

- Analysis of CNN models for detection of COVID-19.

- Analysis of Accuracy and performance of various models utilized.

- The adaptability of the proposed mechanism.

The paper is presented in a total of five folds. Section 1 presents the details of the Socioeconomic and environmental impacts of the COVID-19 pandemic and the relevant research that ended with the gap analysis. The next section 2 is the review of a significant state-of-the-art research article. Section 3 includes the proposed approach with implementation details followed by experimentation section 4 . The next section 5 is the Discussion and Future Work. The last section 6 is the conclusion that infers the knowledge gathered from this study and the future direction that can be followed.

\section{LITERATURE REVIEW}

Since the outbreak, scientists and researchers are actively working to explore the potential of medical radiographs like X-ray scans to detect COVID-19.

Linda et al. [13] developed a framework COVID-Net which makes three predictions on CXR images that they do not have any infection have a non-COVID-19 infection, and that have COVID-19 infection. The dataset utilized, COVIDx, contains 13800 CXR images across 13,725 patients which were generated by combining three publicly available datasets. COVIDx comprises $183 \mathrm{CXR}$ images from 121 COVID-19 confirm patients' instances, the rest of the images are from 8,066 normal patients, and 5,538 non-COVID-19 pneumonia patients. After pre-processing of the ImageNet dataset, COVID-Net was trained on COVIDx with the use of Adam optimizer and tuning the following hyperparameters: learning rate, epochs, batch-size, factor, and patience. The initial COVID-Net model was assessed using Keras and TensorFlow. The quantitative analysis depicts an accuracy of $92.6 \%$, sensitivity of $87.1 \%$, and positive predictive value (PPV) of $96.4 \%$ on COVID-19 cases. The good results show that this CNN model can be used for early detection of COVID-19 and help healthcare works take appropriate measures efficiently. Tulin et al. [14] proposed a framework that provides binary classification as COVID or No-Findings or multi-class classification as COVID or No-Findings or Pneumonia using chest X-ray images which can be used to validate the initial screening of the patients. The authors used the DarkNet model for base architecture and applied Adam optimizer to update weights, cross-entropy loss events, and a learning rate of $3 \mathrm{e}-3$. Outcomes show an accuracy of $98.08 \%$ and $87.02 \%$ for binary and multi-class cases respectively.

Asmaa et al. [15] proposed a model by using a previously designed CNN model, DeTrac (Decompose, Transfer, and Compose), which is capable of handling irregularities in the data repository by decomposing them. DeTrac has three phases, deep feature extraction using pre-training of the CNN of DeTrac and decomposition using class decomposition layer, training using gradient descent, and refinement of the final classification. Data from several hospitals are taken to conduct the study and results show that accuracy is $95.12 \%$, sensitivity $97.91 \%$, specificity $91.87 \%$, and precision $93.36 \%$.

Lawrence et al. [16] created a new model with an amalgam of ResNet-50, VGG16, and a new small CNN. VGG16 comprises 16 layers, and ResNet-50 has 50 layers 
that were used without any changes. The authors replaced the last layer of VGG with a layer containing global average pooling, 65 connected layers with dropout, and a final sigmoid output layer. Ezz et al. [17] demonstrated the result of their study on the public dataset by Dr. Joseph [18] and Dr. Adrian that contains a total of 50 X-ray Images, with 25 normal instances and 25 COVID-19 positive instances. The learning rate applied to the DCNNs used in this study is $\mathrm{e}^{\wedge}-3$, the mini-batch size is 7, and the epochs count is 50. Moreover, they are trained using Stochastic Gradient Descent (SGD) for better convergence and efficient run time. After comparative analysis VGG19 and DenseNet201 architectures are recommended. Muhammad et al. [19] proposed an architecture, named COVID-ResNet by fine-tuning a pre-trained ResNet-50 model that can feed images of size other than that on which it is trained thus increasing efficiency by decreasing epochs count. Therefore, the images are resized and input, then normalized using channel mean and standard deviation as per ImageNet samples. The proposed models showed an accuracy rate of $96.23 \%$ on all classification levels, at epoch's count of 41 , and progressive learning rate optimization starting from 1e-3, and for adam optimizer the mini-batch size was 32.

Asif et al. [20] proposed a system by training a DCNN on a dataset containing 310 CXR images from normal patients, 330 CXR images from patients of bacterial pneumonia, 327 CXR images of viral pneumonia, and 284 CXR images of COVID-19 patients. The proposed framework, called CoroNet, is established through Xception CNN that contains 71 CNN layers pre-trained on ImageNet data. The authors applied transfer learning to avoid overfitting and added a drop-out and 2 connected layers at the end. Moreover, learning of 0.0001 , batch size of 10 , and epoch count of 80 were applied. The results showed an accuracy of $89.5 \%$.

Muhammad et al. [21] presented a CNN model after analyzing four CNN architectures that were pre-trained to segregate images as normal or pneumonia patients. The dataset was used in a mixture of publicly open data repositories containing 190 scans of COVID-19, 1345 scans of viral pneumonia, and 1341 scans of normal CXR. The author used ResNet18 and DenseNet201 for this study to conduct two classification procedures: two and three classifications on models without augmentation and the same using augmentation. Results show best performance on SqueezeNet architecture with an accuracy of $98.3 \%$, specificity of $100 \%$, sensitivity of $96.7 \%$, and precision $-100 \%$ for normal class, and accuracy $=$ $98.3 \%$, sensitivity $=96.7 \%$, specificity $=99 \%$, and precision $=100 \%$ for COVID-19 class. Ali et al. [22] proposed an early detection model to prevent the spread of COVID-19 by taking appropriate measures. The study analyzed pre-trained architectures ResNet50, InceptionV3, and InceptionResNetV2. Results of the study show that ResNet50 performs best with an accuracy of $98 \%$, where InceptionV3 shows the accuracy of $97 \%$ and InceptionResNetV2 shows $87 \%$ accuracy.

Loannis et al. [23] analyzed VGG19, MobileNet V2, Inception, Xception, and Inception ResNet v2. Hyperparameter tuning with Mini batch size 64 and epochs count 10, and 3 class validation and COVID-19 classification showed that performance of MobileNet v2 was highest with 2 class Accuracy $=96.78 \%, 3$ class accuracy $=94.72 \%$ sensitivity $=98.66 \%$, and specificity $=96.46 \%$. Prabira et al. [24] proposed a framework based on ResNet-50 and SVM by conducting training over open datasets available on GitHub, Kaggle, and Open-ai of CXR images, the data is split into 60:20:20 ratio for training, validation, and testing. The study showed the best performance of ResNet50 and SVM with an accuracy of $95.38 \%$, FPR of $95.52 \%$, an F1 score of $91.41 \%$, and MCC and Kappa of 90.76\%. Parnian Afshar et al. [25] presented a framework, COVID-CAPS, that utilizes external data containing 94,323 X-ray images of thorax diseases, containing 4 Convolutional layers and 3 capsule layers, Adam optimizer with learning rate starting from $10^{\wedge}-3$, epochs count 100 and mini-batch size 16 . Results show accuracy $95.7 \%$, sensitivity $90 \%$, specificity $95.8 \%$, and AUC 0.97 . Table 1 shows the state-of-the-art research studies conducted since the start of the year 2020 while Table 2 shows the parametric-based Comparative Analysis of Literature Review.

\begin{tabular}{|c|c|c|c|c|c|c|c|}
\hline Sr\# & $\begin{array}{l}\text { Title and Author [ } \\
\text { 2020] }\end{array}$ & Dataset & Model Details & $\begin{array}{c}\text { Hyperparamet } \\
\text { ers }\end{array}$ & Validations & Classification & $\begin{array}{c}\text { Limitations } \\
\text { and future } \\
\text { work }\end{array}$ \\
\hline 1 & $\begin{array}{l}\text { COVID-Net: A } \\
\text { Tailored Deep } \\
\text { Convolutional } \\
\text { Neural Network } \\
\text { Design for } \\
\text { DetectionofCOVI } \\
\text { D-19CasesfromCh } \\
\text { estX-RayImages by } \\
\text { Linda et al. [1] }\end{array}$ & $\begin{array}{l}\text { COVIDx - } 13800 \\
\text { chest x-ray images, } \\
\text { containing } 183 \\
\text { COVID patient } \\
\text { images. }\end{array}$ & $\begin{array}{l}\text { ImageNet for } \\
\text { pertaining }\end{array}$ & $\begin{array}{c}\text { Learning rate }= \\
2 \mathrm{e}-5, \text { epochs }= \\
22, \text { batch-size }= \\
8, \text { factor }=0.7, \\
\text { patience }=5\end{array}$ & $\begin{array}{l}\text { The initial } \\
\text { evaluation } \\
\text { was done } \\
\text { using Keras } \\
\text { and Tensor } \\
\text { flow, whereas } \\
\text { generative } \\
\text { synthesis was } \\
\text { applied for } \\
\text { building. }\end{array}$ & $\begin{array}{c}3 \text { class } \\
\text { classification } \\
\text { (COVID-19, } \\
\text { Non-COVID- } \\
\text { 19, and } \\
\text { Normal) }\end{array}$ & $\begin{array}{l}\text { Limited } \\
\text { dataset of } \\
\text { COVID-19 } \\
\text { patients } \\
\text { limits the } \\
\text { training } \\
\text { capacity of } \\
\text { Corvid-Net. }\end{array}$ \\
\hline 2 & $\begin{array}{c}\text { Automated } \\
\text { detection of } \\
\text { COVID-19 cases } \\
\text { using deep neural } \\
\text { networks with } \\
\text { X-ray images by } \\
\text { Tulin et al. [2] }\end{array}$ & $\begin{array}{c}\text { Open dataset by } \\
\text { Cohen [J.P. Cohen, } \\
\text { COVID-19 Image } \\
\text { Data Collection, } \\
\text { 2020. } \\
\text { https://github.com/ } \\
\text { ieee } \\
\text { 8023/COVID-ches } \\
\text { t X Ray-dataset] }\end{array}$ & $\begin{array}{c}\text { DarkNet } \\
\text { classifier,17 } \\
\text { convolutional } \\
\text { layers along with } \\
\text { filtering } \\
\text { (BatchNorm) on } \\
\text { each layer, one } \\
\text { flatten, and one } \\
\text { linear layer. }\end{array}$ & $\begin{array}{c}\text { Gradually } \\
\text { increased in } \\
\text { filters to } 8,16, \\
\text { and } 32 \text { an nd } \\
\text { basic DarkNet } \\
\text { parameters as } 19 \\
\text { CNN layers } \\
\text { using Maxpool, } \\
\text { learning rate } \\
\text { 3e-3, Adam } \\
\text { optimizer is } \\
\text { utilized for } \\
\text { updating } \\
\text { weights, and } \\
\text { cross-entropy } \\
\text { loss function. }\end{array}$ & $\begin{array}{l}\text { The model is } \\
\text { assessed by } \\
\text { radiologists }\end{array}$ & $\begin{array}{c}\text { 1.Binary } \\
\text { Class:(COVID } \\
-19 \text { vs } \\
\text { No-Findings) } \\
2 . \\
\text { Multi-Class(C } \\
\text { OVID vs } \\
\text { No-Findings } \\
\text { vs Pneumonia) }\end{array}$ & $\begin{array}{l}\text { Validate on } \\
\text { more } \\
\text { images, } \\
\text { compare the } \\
\text { performanc } \\
\text { e of the } \\
\text { model on } \\
\text { CT images }\end{array}$ \\
\hline 3 & $\begin{array}{c}\text { Classification of } \\
\text { COVID-19 in chest } \\
\text { X-ray images using } \\
\text { DeTraC deep } \\
\text { convolutional } \\
\text { neural network by } \\
\text { Asmaa et al. [3] }\end{array}$ & $\begin{array}{c}\text { Data from various } \\
\text { hospitals and } \\
\text { institutions are } \\
\text { collected. A total of } \\
80 \text { normal CXR } \\
\text { from the Japanese } \\
\text { Society of } \\
\text { Radiological } \\
\text { Technology and } \\
105 \text { CXR and } 11 \\
\text { Coronavirus } \\
\text { samples and SARS } \\
\text { samples were } \\
\text { taken. } \\
\end{array}$ & $\begin{array}{l}\text { Decompose, } \\
\text { Transfer, and } \\
\text { Compose } \\
\text { (DeTraC) } \\
\text { paradigm is used } \\
\text { along with the } \\
\text { ImageNet } \\
\text { pre-trained } \\
\text { ResNet model }\end{array}$ & $\begin{array}{c}\text { Fine-tuning on } \\
\text { parameters is as } \\
\text { follows: } \\
\text { Learning rate } \\
0.0001 \text { for all } \\
\text { except last layer } \\
(0.01), \text { mini } \\
\text { batch size }=64, \\
\text { epochs }=256, \\
\text { weight decay }= \\
0.001 \text { to prevent } \\
\text { overfitting, and } \\
\text { momentum }=0.9\end{array}$ & $\begin{array}{l}\text { The model is } \\
\text { evaluated } \\
\text { through a } \\
\text { confusion } \\
\text { matrix to } \\
\text { analyze its } \\
\text { accuracy, } \\
\text { specificity, } \\
\text { sensitivity, } \\
\text { and precision. }\end{array}$ & $\begin{array}{c}3 \\
\text { classifications } \\
\text { (Normal, } \\
\text { COVID19, } \\
\text { SARS) }\end{array}$ & $\begin{array}{c}\text { Presence of } \\
\text { irregularity } \\
\text { in medical } \\
\text { imaging. }\end{array}$ \\
\hline 4 & $\begin{array}{c}\text { Finding } \\
\text { COVID-19 from } \\
\text { Chest X-rays using } \\
\text { Deep Learning on } \\
\text { a Small Dataset by } \\
\text { Lawrence et al. [4] }\end{array}$ & $\begin{array}{c}\text { Dataset of } 135 \\
\text { CXR images of } \\
\text { COVID-19 } \\
\text { patients, and } 320 \\
\text { CXR images of } \\
\text { viral and bacterial } \\
\text { pneumonia } \\
\text { patients }\end{array}$ & $\begin{array}{l}\text { Pre-trained DNN, } \\
\text { ResNet50 VGG16 } \\
\text { have tuned along } \\
\text { with a new CNN }\end{array}$ & $\begin{array}{c}\text { VGG16 and } \\
\text { ResNet50 by } \\
\text { replacing the } \\
\text { last layer with } \\
\text { global average } \\
\text { pooling, and } 64 \\
\text { connected units. } \\
\text { Learning rate } \\
0.0001 \text { and Max } \\
\text { learning rate = } \\
0.001 \\
\end{array}$ & $\begin{array}{c}10 \text { fold } \\
\text { cross-validati } \\
\text { on using } \\
\text { ResNet50 }\end{array}$ & $\begin{array}{l}\text { on } 3 \text { classes } \\
\text { (COVID-19, } \\
\text { Pneumonia, } \\
\text { and Normal) }\end{array}$ & $\begin{array}{l}\text { The study } \\
\text { has } \\
\text { limitations } \\
\text { due to the } \\
\text { lack of } \\
\text { information } \\
\text { and small } \\
\text { dataset size. }\end{array}$ \\
\hline
\end{tabular}




\begin{tabular}{|c|c|c|c|c|c|c|c|}
\hline 5 & $\begin{array}{l}\text { COVIDX-Net: A } \\
\text { Framework of } \\
\text { Deep Learning } \\
\text { Classifiers to } \\
\text { Diagnose } \\
\text { COVID-19 in } \\
\text { X-Ray Images by } \\
\text { Ezz et al. [5] }\end{array}$ & $\begin{array}{l}50 \text { CXR scans with } \\
25 \text { Coronacirus } \\
\text { patients CXR } \\
\text { scans. }\end{array}$ & $\begin{array}{l}\text { COVIDX-Net } \\
\text { which is an } \\
\text { amalgam of } \\
\text { VGG19, the } \\
\text { second edition of } \\
\text { Google } \\
\text { MobileNet, } \\
\text { DenseNet'-- and } \\
\text { each layer applies } \\
\text { normalization to } \\
\text { classify the } \\
\text { images as COVID } \\
\text { positive or } \\
\text { negative }\end{array}$ & $\begin{array}{l}80: 20 \text { ratio of } \\
\text { X-ray images for } \\
\text { training and } \\
\text { testing. }\end{array}$ & $\begin{array}{l}\text { Cross-validati } \\
\text { on through a } \\
\text { confusion } \\
\text { matrix }\end{array}$ & $\begin{array}{c}\text { binary } \\
\text { classification } \\
\text { (Normal and } \\
\text { COVID-19) }\end{array}$ & $\begin{array}{l}\text { Clinical } \\
\text { testing has } \\
\text { to be } \\
\text { performed. }\end{array}$ \\
\hline 6 & $\begin{array}{l}\text { COVID-ResNet: A } \\
\text { Deep Learning } \\
\text { Framework for } \\
\text { Screening of } \\
\text { COVID19 from } \\
\text { Radiographs by } \\
\text { Muhammad et al. } \\
\text { [6] }\end{array}$ & $\begin{array}{l}\text { Publicly open } \\
\text { COVID-19 dataset } \\
\text { containing 5941 } \\
\text { CXR images } \\
\text { segregated into } \\
\text { no-infection, } \\
\text { bacterial } \\
\text { pneumonia, viral } \\
\text { pneumonia, and } \\
\text { COVID-19 } \\
\text { positive classes. }\end{array}$ & $\begin{array}{l}\text { Radical resizing, } \\
\text { and cyclic } \\
\text { learning rate } \\
\text { tuning on } \\
\text { ResNet50 }\end{array}$ & $\begin{array}{c}\text { Images are } \\
\text { resized to } \\
128 * 128 * 3 \text {, } \\
224 * 224 * 3 \text {, and } \\
229 * 229 * 3 \text {. } \\
\text { Epochs are set to } \\
41\end{array}$ & $\begin{array}{l}\text { A confusion } \\
\text { matrix was } \\
\text { utilized for } \\
\text { validating the } \\
\text { architecture }\end{array}$ & $\begin{array}{c}4 \text { class } \\
\text { classification } \\
\text { (Normal, } \\
\text { Bacterial. } \\
\text { Viral, and } \\
\text { COVID-19) }\end{array}$ & $\begin{array}{l}\text { To declare it } \\
\text { appropriate } \\
\text { for clinical } \\
\text { use, } \\
\text { training on } \\
\text { a larger } \\
\text { dataset is } \\
\text { required. }\end{array}$ \\
\hline 7 & $\begin{array}{l}\text { CoroNet: A Deep } \\
\text { Neural Network for } \\
\text { Detection and } \\
\text { Diagnosis of } \\
\text { Covid-19 from } \\
\text { Chest X-ray } \\
\text { Images by Asif et } \\
\text { al. [7] }\end{array}$ & $\begin{array}{l}\text { A Dataset of a total } \\
\text { of } 1300 \text { images is } \\
\text { generated through } \\
\text { an amalgam of } 2 \\
\text { publicly available } \\
\text { datasets on } \\
\text { github.com by } \\
\text { Joseph and the } \\
\text { other one is } \\
\text { available on } \\
\text { Kaggle.com. }\end{array}$ & $\begin{array}{c}\text { The proposed } \\
\text { model CoroNet is } \\
\text { tested and } \\
\text { validated on an } \\
80 \%: 20 \% \text { ratio. } \\
\text { The base } \\
\text { architecture } \\
\text { utilized is } \\
\text { Xception }\end{array}$ & $\begin{array}{l}\text { Learning rate } \\
\text { applied is } \\
0.0001, \text { batch } \\
\text { size }=10 \text {, and } \\
\text { epochs count }= \\
80 .\end{array}$ & $\begin{array}{l}\text { Validity was } \\
\text { found through } \\
\text { a confusion } \\
\text { matrix }\end{array}$ & $\begin{array}{l}4 \text { types of } \\
\text { infections } \\
\text { (COVID-19, } \\
\text { Normal, } \\
\text { Bacterial } \\
\text { Pneumonia, } \\
\text { and Viral } \\
\text { Pneumonia) }\end{array}$ & $\begin{array}{l}\text { Lack of } \\
\text { training } \\
\text { data }\end{array}$ \\
\hline 8 & $\begin{array}{c}\text { Can AI help in } \\
\text { screening Viral } \\
\text { and COVID-19 } \\
\text { pneumonia? By } \\
\text { Muhammad et al. } \\
{[8]}\end{array}$ & $\begin{array}{c}\text { A dataset was } \\
\text { generated by } \\
\text { mixing } 3 \text { public } \\
\text { data containing } \\
190 \text { COVID-19, } \\
1345 \text { viral } \\
\text { pneumonia, and } \\
1341 \text { normal CXR } \\
\text { scans } \\
\end{array}$ & $\begin{array}{c}\text { The proposed } \\
\text { model employs } \\
\text { tuned ResNet18 } \\
\text { and } \\
\text { DenseNet201, } \\
\text { and SueezeNet. }\end{array}$ & $\begin{array}{c}\text { Momentum } 0.9, \\
\text { mini-batch size } \\
16, \text { and learning } \\
\text { rate } 0.0003 \text { were } \\
\text { utilized. }\end{array}$ & $\begin{array}{l}\text { The validation } \\
\text { is done using } \\
5 \text {-fold } \\
\text { cross-validati } \\
\text { on. }\end{array}$ & $\begin{array}{c}\text { scheme1 } \\
\text { (Normal and } \\
\text { COVID-19), } \\
\text { scheme } 2 \\
\text { (Normal, } \\
\text { Viral, and } \\
\text { COVID-19) }\end{array}$ & - \\
\hline 9 & $\begin{array}{c}\text { Automatic } \\
\text { Detection of } \\
\text { Coronavirus } \\
\text { Disease } \\
\text { (COVID-19) Using } \\
\text { X-ray Images and } \\
\text { Deep } \\
\text { Convolutional } \\
\text { Neural Networks } \\
\text { by Ali et al. [9] }\end{array}$ & $\begin{array}{c}\text { Dataset used } \\
\text { contains } 50 \text { CXR } \\
\text { scans of } \\
\text { Coronavirus } \\
\text { patients from } \\
\text { GitHub by } \\
\text { Dr.Joseph Cohen } \\
\text { and } 50 \text { normal } \\
\text { patients CXR from } \\
\text { the Kaggle } \\
\text { database }\end{array}$ & $\begin{array}{l}\text { Three models are } \\
\text { presented based } \\
\text { on pre-trained } \\
\text { architectures } \\
\text { ResNet50, } \\
\text { InceptionV3, and } \\
\text { InceptionResNet } \\
\text { V2 }\end{array}$ & $\begin{array}{c}\text { Transfer } \\
\text { learning was } \\
\text { used with a } 30 \\
\text { epoch count. }\end{array}$ & $\begin{array}{l}\text { The analysis is } \\
\text { done using } \\
\text { ROC and } \\
\text { 5-fold } \\
\text { cross-validati } \\
\text { on. }\end{array}$ & $\begin{array}{c}\text { Binary } \\
\text { classification } \\
\text { (Normal and } \\
\text { COVID-19) }\end{array}$ & $\begin{array}{l}\text { A limited } \\
\text { number of } \\
\text { the dataset } \\
\text { is the } \\
\text { limitation of } \\
\text { this study }\end{array}$ \\
\hline 10 & $\begin{array}{c}\text { Covid? 19: } \\
\text { automatic } \\
\text { detection } \\
\text { from X? } \\
\text { images utilizing } \\
\text { transfer learning } \\
\text { with convolutional } \\
\text { neural networks by } \\
\text { Ioannis et al. [10] }\end{array}$ & $\begin{array}{c}\text { contains } 1427 \\
\text { CXR scans with } \\
224 \text { Coronavirus } \\
\text { patients \&700 } \\
\text { bacterial } \\
\text { pneumonia, \& } 504 \\
\text { normal } \\
\text { patients, dataset2 } \\
\text { contains } \\
\text { 224images of } \\
\text { COVID-19 } \\
\text { patients, } 714 \\
\text { bacterial,pneumoni } \\
\text { a, \&504 normal } \\
\text { patients }\end{array}$ & $\begin{array}{c}\text { The base } \\
\text { architectures } \\
\text { VGG19, } \\
\text { MobileNet V2, } \\
\text { Inception, } \\
\text { Xception, and } \\
\text { Inception ResNet } \\
\text { v2 are utilized } \\
\text { with dropout } \\
\text { layers and adam } \\
\text { optimizer }\end{array}$ & $\begin{array}{l}\text { Mini batch size } \\
64 \text { and epochs } \\
\text { count } 10\end{array}$ & $\begin{array}{l}\text { Validation is } \\
\text { done using } 3 \\
\text { class accuracy } \\
\text { and } \\
\text { COVID-19 } \\
\text { accuracy and } \\
\text { performance } \\
\text { is measure } \\
\text { using a } \\
\text { confusion } \\
\text { matrix }\end{array}$ & $\begin{array}{l}3 \text { types of } \\
\text { images } \\
\text { (COVID-19, } \\
\text { Pneumonia, } \\
\text { and Normal) }\end{array}$ & $\begin{array}{c}\text { Limited } \\
\text { patient data }\end{array}$ \\
\hline 11 & $\begin{array}{c}\text { Detection of } \\
\text { coronavirus } \\
\text { Disease } \\
\text { (COVID-19) based } \\
\text { on Deep Features } \\
\text { by Prabira et al. } \\
\text { [11] }\end{array}$ & $\begin{array}{l}\text { Data is gathered } \\
\text { from Kaggle, } \\
\text { Github, and Open } \\
\text { ai public } \\
\text { repositories }\end{array}$ & $\begin{array}{c}\text { Deep features of } \\
\text { nine pre-trained } \\
\text { CNN } \\
\text { architectures are } \\
\text { extracted and } \\
\text { passed to SVM } \\
\text { classifier for } \\
\text { analysis }\end{array}$ & $\begin{array}{c}100 \text { individual } \\
\text { simulations are } \\
\text { applied }\end{array}$ & $\begin{array}{c}\text { Statistical } \\
\text { analysis is } \\
\text { done upon the } \\
\text { accuracy, } \\
\text { sensitivity, } \\
\text { and specificity } \\
\text { of the } \\
\text { architectures } \\
\text { under } \\
\text { consideration }\end{array}$ & $\begin{array}{c}\text { binary } \\
\text { classification } \\
\text { (COVID-19+ } \\
\text { and } \\
\text { COVID-19 -) }\end{array}$ & $\begin{array}{c}\text { Dataset is } \\
\text { limited }\end{array}$ \\
\hline 12 & $\begin{array}{c}\text { COVID-CAPS: A } \\
\text { Capsule } \\
\text { Network-based } \\
\text { Framework for } \\
\text { Identification of } \\
\text { COVID-19 cases } \\
\text { from X-ray Images } \\
\text { by Parnian Afshar } \\
\text { et al. [12] }\end{array}$ & $\begin{array}{c}\text { External data } \\
\text { containing 94,323 } \\
\text { Xray images of } \\
\text { thorax diseases }\end{array}$ & $\begin{array}{c}\text { The proposed } \\
\text { model, } \\
\text { COVID-CAPS } \\
\text { contains } 4 \\
\text { Convolutional } \\
\text { layers and } 3 \\
\text { capsule layers }\end{array}$ & $\begin{array}{l}\text { Adam optimizer } \\
\text { with learning } \\
\text { rate starting } \\
\text { from } 10^{\wedge}-3, \\
\text { epochs count } \\
100 \text { and } \\
\text { mini-batch size } \\
16 \text { is used }\end{array}$ & $\begin{array}{l}\text { Confusions } \\
\text { matrix is used } \\
\text { to validate the } \\
\text { performance }\end{array}$ & - & $\begin{array}{l}\text { Lack of } \\
\text { dataset is } \\
\text { the } \\
\text { limitation }\end{array}$ \\
\hline
\end{tabular}


Table 2: Parametric Comparative Analysis of Literature Review

\begin{tabular}{|c|c|c|c|c|c|c|}
\hline No & Title and Author [ 2020] & Accuracy & Sensitivity & $\begin{array}{c}\text { Specificit } \\
\mathbf{y}\end{array}$ & Precision & Other Parameters \\
\hline 1 & $\begin{array}{l}\text { COVID-Net: A Tailored Deep } \\
\text { Convolutional Neural Network } \\
\text { Design for Detection of } \\
\text { COVID-19 Cases from Chest } \\
\text { X-Ray Images by Linda et al. [1] }\end{array}$ & $92.6 \%$ & $87.1 \%$ & - & - & Positive predictive value $96.4 \%$ \\
\hline 2 & $\begin{array}{l}\text { Automated detection of } \\
\text { COVID-19 cases using deep } \\
\text { neural networks with X-ray } \\
\text { images by Tulin et al. [2] }\end{array}$ & $\begin{array}{c}\text { Accuracy= } \\
98.08 \% \text { and } \\
87.02 \% \text { for } \\
\text { binary and } \\
\text { multi-class } \\
\text { cases } \\
\text { respectively. }\end{array}$ & - & - & - & - \\
\hline 3 & $\begin{array}{c}\text { Classification of COVID-19 in } \\
\text { chest X-ray images using } \\
\text { DeTraC deep convolutional } \\
\text { neural network by Asmaa et al. } \\
\text { [3] }\end{array}$ & $\begin{array}{l}\text { Accuracy } \\
=95.12 \%\end{array}$ & $97.91 \%$, & $91.87 \%$ & $\begin{array}{c}\text { Precision(fracti } \\
\text { on of relevant } \\
\text { instances } \\
\text { among the } \\
\text { retrieved } \\
\text { instances) } \\
93.36 \% \\
\end{array}$ & - \\
\hline 4 & $\begin{array}{c}\text { Finding COVID-19 from Chest } \\
\text { X-rays using Deep Learning on } \\
\text { a Small Dataset by Lawrence et } \\
\text { al. [4] }\end{array}$ & $\begin{array}{c}\text { Accuracy = } \\
91.24 \%\end{array}$ & $\begin{array}{c}\text { True } \\
\text { positive rate } \\
=0.7879\end{array}$ & - & - & $\begin{array}{c}\text { False-positive rate(number of } \\
\text { negative events wrongly categorized } \\
\text { as positive) } 6.88 \% \text { and AUC (Area } \\
\text { Under Curve) }=0.94\end{array}$ \\
\hline 5 & $\begin{array}{c}\text { COVIDX-Net: A Framework } \\
\text { of Deep Learning Classifiers to } \\
\text { Diagnose COVID-19 in X-Ray } \\
\text { Images by Ezz et al. [5] }\end{array}$ & - & - & - & - & $\begin{array}{l}\text { VGG19 and DenseNet } \\
\text { demonstrate } 0.89 \text { and } 0.91 \mathrm{f} 1 \\
\text { classification scores (balance } \\
\text { between the precision and the } \\
\text { recall). Whereas, Inception } \\
\text { showed inappropriate results with } \\
0.67 \mathrm{f} 1 \text { scores for normal patients } \\
\text { and } 0.00 \text { for Coronavirus patients. }\end{array}$ \\
\hline 6 & $\begin{array}{c}\text { COVID-ResNet: A Deep } \\
\text { Learning Framework for } \\
\text { Screening of COVID19 from } \\
\text { Radiographs by Muhammad et } \\
\text { al. [6] }\end{array}$ & $\begin{array}{l}\text { Accuracy } \\
\text { rate } \\
\text { achieved } \\
\text { in } 96.23 \%\end{array}$ & - & - & - & - \\
\hline 7 & $\begin{array}{c}\text { CoroNet: A Deep Neural } \\
\text { Network for Detection and } \\
\text { Diagnosis of Covid-19 from } \\
\text { Chest X-ray Images by Asif et } \\
\text { al. [7] }\end{array}$ & $\begin{array}{l}\text { Accuracy } \\
=89.5 \%\end{array}$ & $\begin{array}{c}\text { Recall rate }= \\
100 \%\end{array}$ & - & $\begin{array}{c}\text { Precision = } \\
97 \%\end{array}$ & - \\
\hline 8 & $\begin{array}{c}\text { Can AI help in screening Viral } \\
\text { and COVID-19 pneumonia? } \\
\text { By Muhammad et al. [8] }\end{array}$ & $\begin{array}{l}\text { Accuracy } \\
=98.3 \% \\
\quad \text { for } \\
\text { scheme } 1, \\
\quad \text { and } \\
\text { accuracy = } \\
98.3 \%, \text { for } \\
\text { scheme } 2\end{array}$ & $\begin{array}{c}\text { 96.7\% for } \\
\text { scheme } 1 \\
\text { And } 96.7 \% \\
\text { for scheme } 2\end{array}$ & $\begin{array}{c}100 \% \\
\text { for } \\
\text { scheme1 } \\
\& 99 \% \\
\text { for } \\
\text { scheme } \\
2\end{array}$ & $\begin{array}{c}\text { precision - } \\
100 \% \text { for } \\
\text { scheme1 \& } \\
\text { precision }= \\
100 \% \text { for } \\
\text { scheme } 2\end{array}$ & - \\
\hline 9 & $\begin{array}{l}\text { Automatic Detection of } \\
\text { Coronavirus Disease } \\
\text { (COVID-19) Using X-ray } \\
\text { Images and Deep } \\
\text { Convolutional Neural } \\
\text { Networks by Ali et al. [9] }\end{array}$ & $\begin{array}{c}\text { Results } \\
\text { show that } \\
\text { ResNet50 } \\
\text { performs } \\
\text { best with } \\
\text { accuracy } \\
98 \%, \\
\text { where } \\
\text { Inception } \\
\text { V3 shows } \\
\text { accuracy } \\
\text { 97\% and } \\
\text { InceptionR } \\
\text { esNetV2 } \\
\text { shows } 87 \% \\
\text { accuracy }\end{array}$ & - & - & - & - \\
\hline 10 & $\begin{array}{c}\text { Covid? 19: detection } \\
\text { from X? ray images by transfer } \\
\text { learning with convolutional } \\
\text { neural networks. [10] }\end{array}$ & $\begin{array}{c}\text { MobileNet } \\
\text { v2 } \\
\text { demonstrat } \\
\text { es high } 2 \\
\text { class } \\
\text { Accuracy } \\
=96.78 \%, \\
3 \text { class } \\
\text { accuracy = } \\
94.72 \% \\
\end{array}$ & $\begin{array}{c}98.66 \% \text {, on } \\
3 \text { class }\end{array}$ & $\begin{array}{l}96.46 \% \\
\text { on } 3 \\
\text { class }\end{array}$ & - & - \\
\hline 11 & $\begin{array}{c}\text { Detection of coronavirus } \\
\text { Disease (COVID-19) based on } \\
\text { Deep Features by Prabira et al. } \\
\text { [11] }\end{array}$ & $\begin{array}{l}\text { ResNet50 } \\
\text { and SVM } \\
\text { with an } \\
\text { accuracy of } \\
95.38 \%\end{array}$ & - & - & - & $\begin{array}{l}\text { FPR score } 95.52 \%, \text { and F1 score } \\
91.41 \%, \text { and MCC(quality of } \\
\text { binary classifications) and } \\
\text { Kappa(how closely the instances } \\
\text { classified matched the data labeled } \\
\text { as ground truth) of } 90.76 \%\end{array}$ \\
\hline 12 & $\begin{array}{l}\text { COVID-CAPS: A Capsule } \\
\text { Network-based Framework for } \\
\text { Identification of COVID-19 } \\
\text { cases from X-ray Images by } \\
\text { Parnian Afshar et al. [12] }\end{array}$ & $\begin{array}{c}\text { Results } \\
\text { show an } \\
\text { accuracy of } \\
98.3 \% \text { for } \\
\text { pre-trained } \\
\text { COVID-C } \\
\text { APS } \\
\end{array}$ & $80 \%$ & $98.6 \%$ & - & - \\
\hline
\end{tabular}


A study of literature shows that the SqueezeNet model demonstrates better performance for COVID-19 detection in X-ray Images using transfer learning. In the next section, we propose a model to analyze the performance of the model on a smaller dataset in comparison to a larger dataset.

\section{PROPOSED APPROACH}

The literature analysis conducted in this study showed that most of the researchers proposed architectures based on the previous convolutional neural networks used in medical imaging. Explicitly, the process of Transfer Learning was adopted. Transfer learning is a method that utilizes a prototype trained earlier for a particular setting and modifies it, by replacing its final layers, to improve it to be used in another setting. Then, the model is trained and its accuracy is assessed. This is the most suitable technique in the current scenario where although a large number of patients are suffering from COVID-19, the publicly available data set is very limited, thus causing hindrance in research work. Figure 1 shows the way transfer learning is used to develop custom architectures from pre-trained models like AlexNet [26], SqueezeNet [27], and VGG.

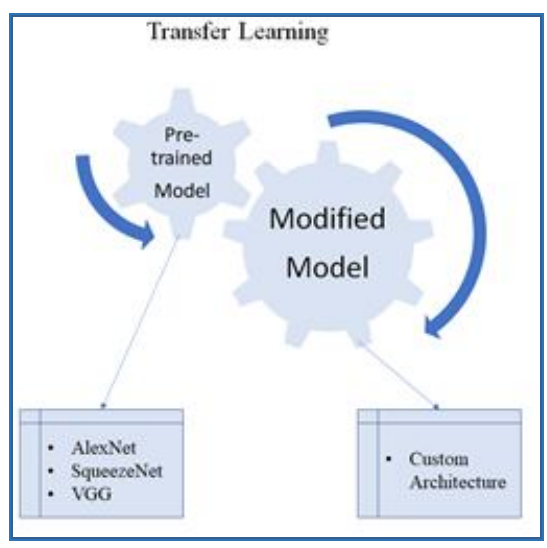

Figure 1: Process of Transfer Learning used for Pre-trained Convolutional Neural Networks

For this purpose, pre-trained models utilizing ImageNet [28], that is a data repository generated for utilization in visual object identification software research, classifier, DarkNet [29], that is an open-source neural network framework to train neural networks, classifier, ResNet [30] model, also known as Residual Networks that allows training deep neural networks with $150+$ layers, VGG, also known as Visual Geometry Group model that achieves around $92.7 \%$ after training on millions of images, and SqueezeNet, that is a Deep Neural Network designed for computer vision in 2016 were utilized. Results showed that SqueezeNet outperforms other models.

SqueezeNet [27] was developed by a group of researchers from the University of California, Berkeley, and Stanford University, at Deep Scale. The main aim was to achieve ease in transmission over computer networks, high suitability to deploy on hardware having limited memory, and the creation of a smaller neural network. This resulted in a model that has 50 times fewer parameters with the same accuracy as AlexNet and around $0.5 \mathrm{MB}$ model size. SqueezeNet is designed to switch $3 \times 3$ filters with $1 \times 1$ filters as they contain 9 times fewer parameters. But it is not possible to create a CNN with a $1 \times 1$ filter, thus, $3 \times 3$ filters are used in the fire module of the model. The fire module is the base for building this model, it contains two layers: a squeeze layer, also known as a bottle-neck layer, and an expand layer, each containing convolutional filters which can be decided according to the framework being developed. Moreover, with the help of the fire module, delayed down-sampling is utilized on the spatially larger activation map of the CNN, so that important features are not lost from the dimension of the feature map earlier in the network additionally, similar to network compression which was traditionally applied to larger networks like AlexNet and VGG only. Figure 2 shows the layers of the fire module of SqueezeNet which is a notable part of the model also referred to as the building block of the CNN. It consists of a squeeze layer and an expand layer as shown in the figure.

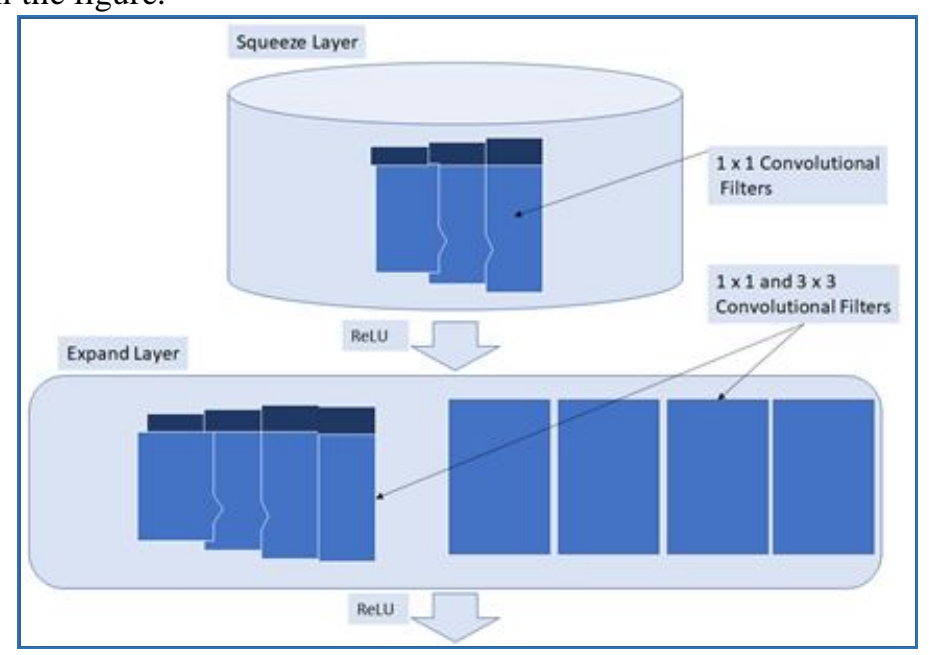

Figure 2: Fire Module of SqueezeNet

Deep neural networks usually perform best in larger datasets rather than smaller ones. For this research, the transfer learning model is analyzed on two sizes of datasets. First, a dataset is generated, by an amalgam of three public datasets, containing a total of 219 Covid19 Images, 1341 Normal Chest Xray images, and 1345 Pneumonia Images. Second, a training set of 2600 images of each category was used to train and validate four state-of-the-art pre-trained deep CNNs. The assessment was based on 2 schemes (normal and COVID-19 pneumonia; and normal, viral pneumonia, and COVID-19 pneumonia). The results of experimentation showed an accuracy of $95.11 \%$
Due to the novelty of COVID-19, there is a lack of enough datasets, thus we generated another dataset through an amalgam of publicly available datasets and preprocessed.

First, a dataset was generated, by an amalgam of three public datasets selected from github.com and Kaggle.com [31] [32]. The new images were resized to 1024x1024 and converted from jpg to png, then merged with the images of the older dataset. The new dataset which we obtained by following these steps included a total of 795 Covid19 images, 1341 Normal Chest X-Ray Images, and 5618 Pneumonia images. The training set, which is the set of pairs of input patterns with corresponding desired output patterns, and the Test set, which is a set of examples used only to assess the performance of a fully specified classifier, were separated. Details of the assessment are provided in the next section of experimentation.

\section{EXPERIMENTATION}

The assessment is based on 2 schemes (normal and COVID-19 pneumonia; and normal, viral pneumonia, and COVID-19 pneumonia). The pre-trained SqueezeNet model was selected for experimentation. This model showed high accuracy in state-of-the-art CNN models for detecting COVID-19. Experimentation was conducted on the training and test set. Finally, the performance was evaluated using the performance metrics known as accuracy defined as the number of correct predictions made by the model.

The experiment was conducted following 650 iterations and in 5 training cycles denoted as epochs where 130 iterations per epoch are used for validation. A maximum of 650 iterations is used. A single GPU machine is used for experimentation purposes. The training result of 650 iterations is shown in Figure 3.

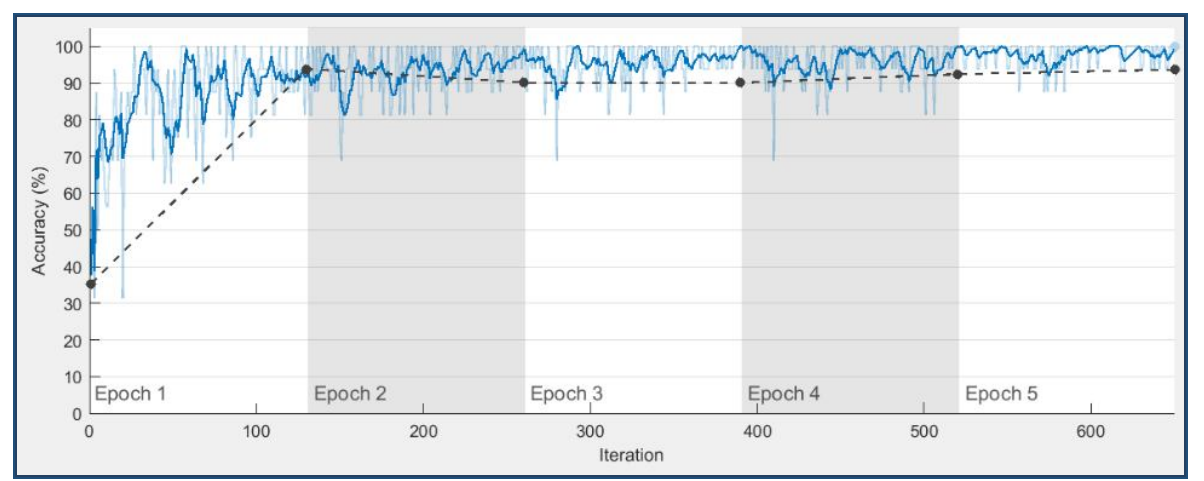

Figure 3: Training Result of 650 iterations

The experimentation showed an accuracy of $95.11 \%$ that was obtained on a dataset of 2905 images and an accuracy of $96.07 \%$ was obtained on an augmented dataset of 9337 images to detect Covid-19 in X-ray images. In the image input layer of [227 $2273]$, the Input size is used with an average image of [ $1 * 1 * 3$ single]. The zero center normalization method was used. Accuracy results are shown graphically in Figure 4.

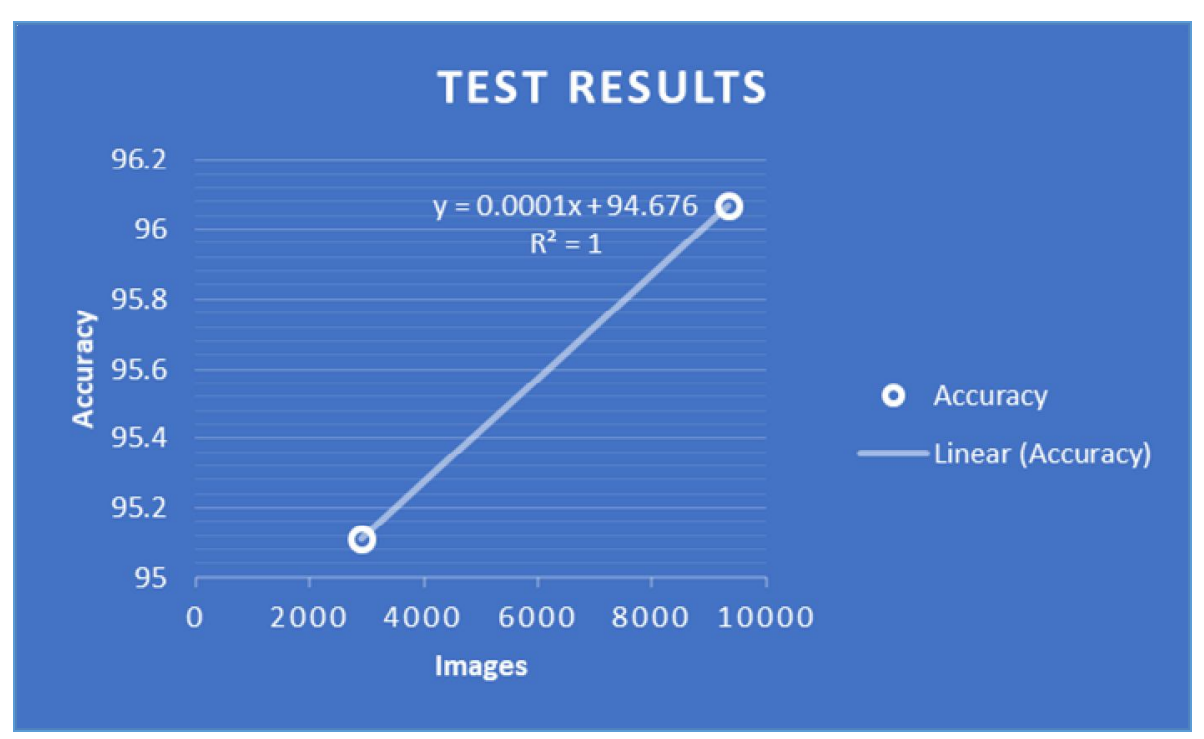

Figure 4: Accuracy Results

\section{DISCUSSION AND FUTURE WORK}

The advancement in software applications is leading to the exploration of various areas including health care, manufacturing industry, and so on [33]-[42]. The speedy advancement of the strong branches of artificial intelligence in the field of medical imaging and the improving performance of deep learning approaches in the detection of COVID-19 has opened a gate for many more fruitful pieces of research to sprout. As shown in the study above, largely successful attempts have been made to increase efficiency and accuracy in the method of COVID-19 detection. Our research study has shown that the deep learning model generated by applying transfer learning on SqueezeNet shows high accuracy in the detection of COVID-19. Furthermore, our study shows that increasing the size of the training dataset increases the accuracy of the convolutional neural network.

The detection of COVID-19 would enhance the safety of health providers by minimizing the direct contact of health practitioners with the patients, early detection of COVID-19 in patients will allow them to be quarantined without delay and reduce the chances of exposure of people around them. Moreover, the cost of expensive kits used in the PCR methods for the detection of this disease can be reduced by utilizing the commonly available $\mathrm{X}$-ray scans. However, there are many positive and negative aspects of this field that needs to be addressed in the future. Some of them are stated as follows:

First, the amount of trust put on the artificial intelligence techniques for better image acquisition with the least involvement of healthcare professionals needs to be enhanced utilizing a more advanced technique which would generate better image quality with the least radiation exposure of the patients through tuning the $\mathrm{x}$-ray parameters. 
Second, the probability of ambiguity of the medical images by depicting radiological signs is still high due to the limitation of processed data therefore, overfitting of the model conclusions may occur. Therefore, more data needs to be acquired to increase the quality of the results produced.

Third, deep learning is proving to be a prevailing approach towards medical imaging by its performance of COVID-19 detection. However, these models require a vast amount of data that is not easily publicly available which is why transfer learning is used. Therefore, efforts need to be made to publish completely and accurately labeled data for training deep learning models and broadening the scope of this field.

\section{CONCLUSION}

COVID-19 has implied immense damage to medical health and economic bodies around the globe. Even with the discovery of the vaccine, the spread has been unstoppable so far due to different reasons including the generation of several deadly variants of coronavirus. To stop the spread of this disease, we need to explore the methods of early detection of COVID-19 in potential patients. This study demonstrates the significance of deep learning techniques by utilizing convolutional neural networks for the diagnosis of COVID-19. The analysis shows that transfer learning is a decent approach for detecting COVID-19 in X-ray images and classifying them based on Normal, Viral Pneumonia, and COVID-19 classes. Among the state-of-the-art substantial research work, SqueezeNet demonstrates the highest accuracy for this classification. In this research, we evaluated a SqueezeNet model on a smaller dataset consisting of 2905 images that showed an accuracy of $95.11 \%$ and a larger dataset consisting of 9337 images that showed an accuracy of $96.07 \%$. The improvement in the accuracy level shows that deep learning models perform better if the training datasets are larger

The overall benefit of deep learning models in this scenario is worth appreciating, where it can not only save many human lives by controlling the spread of the disease through early detection but also help in the reduction of extra costs by using the existing machines like X-ray machines available in the frontline hospitals globally. Therefore, it is important to blend the imaging data and the clinical manifestations to solve the issues faced during this pandemic by enhancing patients' screening, disease detection, and optimizing resources. As a result, we conclude that computational intelligence is a greatly significant approach to perform highly accurate and optimized identification and screening of COVID-19 patients.

\section{REFERENCES}

1. Africa, A.D.M., Asuncion, F.X., Tiberio, J.L. and Munchua, R.M.F.A., Sensor-based traffic control network with neural network based control system. International Journal of Advanced Trends in Computer Science and Engineering, 8(4), p.983,2019.

2. W. Guan et al., 'Clinical Characteristics of Coronavirus Disease 2019 in China', N Engl J Med, vol. 382, no. 18, pp. 1708-1720, Apr. 2020, doi: 10.1056/NEJMoa2002032

3. C. Huang et al., 'Clinical features of patients infected with 2019 novel coronavirus in Wuhan, China', The Lancet, vol. 395, no. 10223, pp. 497-506, Feb. 2020, doi: 10.1016/S0140-6736(20)30183-5.

4. A. A. Farid, G. I. Selim, and H. A. A. Khater, 'A Novel Approach of CT Images Feature Analysis and Prediction to Screen for Corona Virus Disease (COVID-19)', IJSER, vol. 11, no. 03, pp. 1141-1149, Mar. 2020, doi: 10.14299/ijser.2020.03.02.

5. T. Ai et al., 'Correlation of Chest CT and RT-PCR Testing in Coronavirus Disease 2019 (COVID-19) in China: A Report of 1014 Cases', Radiology, p. 200642, Feb. 2020, doi: 10.1148/radiol.2020200642.

6. R. M. Pereira, D. Bertolini, L. O. Teixeira, C. N. Silla, and Y. M. G. Costa, 'COVID-19 identification in chest X-ray images on flat and hierarchical classification scenarios', Computer Methods and Programs in Biomedicine, $\mathrm{p}$. 105532, May 2020, doi: 10.1016/j.cmpb.2020.105532.

7. Mundher Mohammed Taresh, Ningbo Zhu, Talal Ahmed Ali Ali, Asaad Shakir Hameed, Modhi Lafta Mutar, "Transfer Learning to Detect COVID-19 Automatically from X-Ray Images Using Convolutional Neural Networks", International Journal of Biomedical Imaging, vol. 2021, Article ID 8828404, 9 pages, 2021.

8. Sharifrazi D, Alizadehsani R, Roshanzamir M, et al. Fusion of convolution neural network, support vector machine and Sobel filter for accurate detection of COVID-19 patients using X-ray images. Biomed Signal Process Control. 2021;68:102622. doi:10.1016/j.bspc.2021.102622.

9. Qi, X., Brown, L.G., Foran, D.J. et al. Chest X-ray image phase features for improved diagnosis of COVID-19 using convolutional neural network. Int J CARS 16, 197-206 (2021). https://doi.org/10.1007/s11548-020-02305-w.

10. A. Becker, 'Artificial intelligence in medicine: What is it doing for us today?', Health Policy and Technology, vol. 8, no. 2, pp. 198-205, Jun. 2019, doi: 10.1016/j.hlpt.2019.03.004.

11. S. Lawrence, C. L. Giles, Ah Chung Tsoi, and A. D. Back, 'Face recognition: a convolutional neural-network approach', IEEE Trans. Neural Netw., vol. 8, no. 1, pp. 98-113, Jan. 1997, doi: 10.1109/72.554195.

12. Y. Kim, 'Convolutional Neural Networks for Sentence Classification', arXiv:1408.5882 [cs], Sep. 2014, Accessed: Jun. 10, 2020. [Online]. Available: http://arxiv.org/abs/1408.5882.

13. L. Wang and A. Wong, 'COVID-Net: A Tailored Deep Convolutional Neural Network Design for Detection of COVID-19 Cases from Chest X-Ray Images', arXiv:2003.09871 [cs, eess], May 2020, Accessed: May 22, 2020. [Online]. Available: http://arxiv.org/abs/2003.09871.

14. T. Ozturk, M. Talo, E. A. Yildirim, U. B. Baloglu, O. Yildirim, and U. Rajendra Acharya, 'Automated detection of COVID-19 cases using deep neural networks with X-ray images', Computers in Biology and Medicine, vol. 121, p. 103792, Jun. 2020, doi: 10.1016/j.compbiomed.2020.103792.
15. A. Abbas, M. M. Abdelsamea, and M. M. Gaber, 'Classification of COVID-19 in chest X-ray images using DeTraC deep convolutional neural network', arXiv:2003.13815 [cs, eess, stat], May 2020, Accessed: May 22, 2020. [Online]. Available: http://arxiv.org/abs/2003.13815

16. L. O. Hall, R. Paul, D. B. Goldgof, and G. M. Goldgof, 'Finding Covid-19 from Chest X-rays using Deep Learning on a Small Dataset' arXiv:2004.02060 [cs, eess], May 2020, Accessed: May 22, 2020. [Online]. Available: http://arxiv.org/abs/2004.02060.

17. E. E.-D. Hemdan, M. A. Shouman, and M. E. Karar, 'COVIDX-Net: A Framework of Deep Learning Classifiers to Diagnose COVID-19 in X-Ray Images', arXiv:2003.11055 [cs, eess], Mar. 2020, Accessed: May 22, 2020 [Online]. Available: http://arxiv.org/abs/2003.11055.

18. https://www.pyimagesearch.com/category/medical/,https://github.com/ieee80 23/covid-chestxray-dataset

19. M. Farooq and A. Hafeez, 'COVID-ResNet: A Deep Learning Framework for Screening of COVID19 from Radiographs', arXiv:2003.14395 [cs, eess] Mar. 2020, Accessed: May 22, 2020. [Online]. Available: http://arxiv.org/abs/2003.14395.

20. A. I. Khan, J. L. Shah, and M. Bhat, 'CoroNet: A Deep Neural Network for Detection and Diagnosis of Covid-19 from Chest X-ray Images', arXiv:2004.04931 [cs, eess, stat], Apr. 2020, Accessed: May 22, 2020 [Online]. Available: http://arxiv.org/abs/2004.04931.

21. M. E. H. Chowdhury et al., 'Can AI help in screening Viral and COVID-19 pneumonia?', arXiv:2003.13145 [cs], Apr. 2020, Accessed: May 22, 2020 [Online]. Available: http://arxiv.org/abs/2003.13145.

22. A. Narin, C. Kaya, and Z. Pamuk, 'Automatic Detection of Coronavirus Disease (COVID-19) Using X-ray Images and Deep Convolutional Neura Networks', arXiv:2003.10849 [cs, eess], Mar. 2020, Accessed: May 22, 2020. [Online]. Available: http://arxiv.org/abs/2003.10849.

23. I. D. Apostolopoulos and T. A. Mpesiana, 'Covid-19: automatic detection from X-ray images utilizing transfer learning with convolutional neura networks', Phys Eng Sci Med, Apr. 2020, doi: 10.1007/s13246-020-00865-4.

24. P. K. Sethy and S. K. Behera, 'Detection of Coronavirus Disease (COVID-19) Based on Deep Features', ENGINEERING, preprint, Mar. 2020. doi 10.20944/preprints202003.0300.v1

25. P. Afshar, S. Heidarian, F. Naderkhani, A. Oikonomou, K. N. Plataniotis, and A. Mohammadi, 'COVID-CAPS: A Capsule Network-based Framework for Identification of COVID-19 cases from X-ray Images', arXiv:2004.02696 [cs, eess], Apr. 2020, Accessed: May 22, 2020. [Online] Available: http://arxiv.org/abs/2004.02696.

26. M. Z. Alom et al., 'The History Began from AlexNet: A Comprehensive Survey on Deep Learning Approaches', arXiv:1803.01164 [cs], Sep. 2018 Accessed: Jun. 29, 2020. [Online]. Available: http://arxiv.org/abs/1803.01164.

27. F. N. Iandola, S. Han, M. W. Moskewicz, K. Ashraf, W. J. Dally, and K. Keutzer, 'SqueezeNet: AlexNet-level accuracy with 50x fewer parameters and <0.5MB model size', arXiv:1602.07360 [cs], Nov. 2016, Accessed: Jun. 29, 2020. [Online]. Available: http://arxiv.org/abs/1602.07360.

28. J. Deng, W. Dong, R. Socher, L.-J. Li, Kai Li, and Li Fei-Fei, 'ImageNet: A large-scale hierarchical image database', in 2009 IEEE Conference on Computer Vision and Pattern Recognition, Miami, FL, Jun. 2009, pp. 248-255, doi: 10.1109/CVPR.2009.5206848

29. Hendry and R.-C. Chen, 'Automatic License Plate Recognition via sliding-window darknet-YOLO deep learning', Image and Vision Computing, vol. 87, pp. 47-56, Jul. 2019, doi: 10.1016/j.imavis.2019.04.007.

30. S. Targ, D. Almeida, and K. Lyman, 'Resnet in Resnet: Generalizing Residual Architectures', arXiv:1603.08029 [cs, stat], Mar. 2016, Accessed: Jun. 29, 2020. [Online]. Available: http://arxiv.org/abs/1603.08029.

31. https://www.kaggle.com/tawsifurrahman/covid19-radiography-database

32. https://www.kaggle.com/bachrr/covid-chest-xray

33. M. R. Naqvi, M. Aslam, M. W. Iqbal, S. Khuram Shahzad, M. Malik and M. U. Tahir, 'Study of Block Chain and its Impact on Internet of Health Things (IoHT):Challenges and Opportunities', 2020 International Congress on Human-Computer Interaction, Optimization and Robotic Applications (HORA), 2020, pp. 1-6, doi: 10.1109/HORA49412.2020.9152846.

34. Naqvi, Muhammad Raza. 'Low power network on chip architectures: A survey.' Computer Science and Information Technologies 2.3 (2021): 158-168.

35. Malik, Marium, Muhammad Imran Tariq, Maira Kamran, and Muhammad Raza Naqvi. 'Artificial Intelligence in Medicine.' In Advances in Smart Vehicular Technology, Transportation, Communication and Applications, pp 159-170. Springer, Singapore, 2021.M. R. Naqvi, M. W. Iqbal, S. K. Shahzad, I. Tariq, M. Malik, F. Ehsan, et al., 'A Concurrence Study on Interoperability Issues in IoT and Decision Making Based Model on Data and Services being used during Inter-Operability', LGURJCSIT, vol. 4, no 4, pp. 73-85, 2020.

36. M. Malik, M. A. Jaffar and M. R. Naqvi, 'Comparison of Brain Tumor Detection in MRI Images Using Straightforward Image Processing Techniques and Deep Learning Techniques, 2021 3rd International Congress on Human-Computer Interaction, Optimization and Robotic Applications (HORA), 2021, pp. 1-6, doi: 10.1109/HORA52670.2021.9461328.

37. Niazi, Adan Khan, Maira Kamran, Zulfiqar Tariq, Marium Malik, Khadija Ilyas, and Muhammad Tahir Zaman. 'Prediction of exercise pattern using compression of different algorithms.'

38. Niazi, Adan Khan, Maira Kamran, Khadija Ilyas, Marium Malik, Zulfiqar Tariq, and Muhammad Tahir Zaman. 'Comparative Sentiment analysis of product review using machine learning and deep learning algorithm.'

39. Niazi, Adan Khan, Maira Kamran, Zulfiqar Tariq, Marium Malik, Khadija Ilyas, and Muhammad Tahir Zaman. 'Prediction of Machine Effected Factor via Statistics Analysis using Data mining.'

40. Niazi, Adan Khan, Maira Kamran, Zulfiqar Tariq, Marium Malik, Khadija Ilyas, and Muhammad Tahir Zaman. 'Statistics Industrial Perceptive Sales Analysis using Data mining.' 
41. Tariq, Zulfiqar, Maira Kamran, Adan Khan Niazi, Marium Malik, Khadija Ilyas, and Muhammad Tahir Zaman. 'Analysing Covid-19 Total, Active,

Recovered cases and Deaths in Pakistan using Time Series Decomposition.'

42. Shahzad, Syed Khuram, Daniyal Ahmed, Muhammad Raza Naqvi, Muhammad Tahir Mushtaq, Muhammad Waseem Iqbal, and Farrukh Munir. 'Ontology Driven Smart Health Service Integration.' Computer Methods and Programs in Biomedicine 207 (2021): 106146. 